\title{
The effect of slow passage in the pulse-pumped quantum dot laser
}

\author{
G.S. Sokolovskii ${ }^{a}$, M. Abu Saa ${ }^{\text {b,c }}$, J. Danckaert ${ }^{b}$, V.V. Dudelev ${ }^{a}$, A.G. Deryagin ${ }^{a}$, I.I. Novikov ${ }^{a}$, \\ M.V. Maximov", A.E. Zhukov', V.M. Ustinov', ${ }^{\mathrm{a}}$, V.I. Kuchinskii ${ }^{\mathrm{a}, \mathrm{e}}$, W. Sibbett ${ }^{\mathrm{f}}$, E.U. Rafailov ${ }^{\mathrm{g}}$, \\ E.A. Viktorov,i, T. Erneux ${ }^{\mathrm{i}}$ \\ ${ }^{a}$ Ioffe Physical-Technical Institute, St. Petersburg, Russia \\ ${ }^{\mathrm{b}}$ Applied Physics Research Group (APHY), Vrije Universiteit Brussel, Brussels, Belgium \\ ${ }^{c}$ Physics Department, Arab American University, Jenin, Palestinee \\ ${ }^{d}$ Academic University, St. Petersburg, Russia \\ e St.Petersburg State Electrotechnical University "LETI", St. Petersburg, Russia \\ ${ }^{\mathrm{f}}$ School of Physics and Astronomy, University of St. Andrews, St. Andrews, UK \\ ${ }^{\mathrm{g}}$ Aston Institute of Photonic Technologies, Aston University, Birmingham, UK \\ ${ }^{\mathrm{h}}$ National Research University of Information Technologies, Mechanics and Optics, \\ St. Petersburg, Russia \\ i Optique Nonlineaire Theorique, Universite Libre de Bruxelles, Brussel, Belgium
}

\begin{abstract}
In recent years, quantum-dot (QD) semiconductor lasers attract significant interest in many practical applications due to their advantages such as high-power pulse generation because to the high gain efficiency. In this work, the pulse shape of an electrically pumped QD-laser under high current is analyzed. We find that the slow rise time of the pulsed pump may significantly affect the high intensity output pulse. It results in sharp power dropouts and deformation of the pulse profile. We address the effect to dynamical change of the phase-amplitude coupling in the proximity of the excited state (ES) threshold. Under 30ns pulse pumping, the output pulse shape strongly depends on pumping amplitude. At lower currents, which correspond to lasing in the ground state (GS), the pulse shape mimics that of the pump pulse. However, at higher currents the pulse shape becomes progressively unstable. The instability is greatest when in proximity to the secondary threshold which corresponds to the beginning of the ES lasing. After the slow rise stage, the output power sharply drops out. It is followed by a long-time power-off stage and large-scale amplitude fluctuations. We explain these observations by the dynamical change of the alpha-factor in the QD-laser and reveal the role of the slowly rising pumping processes in the pulse shaping and power dropouts at higher currents. The modeling is in very good agreement with the experimental observations.
\end{abstract}

Keywords: Semiconductor lasers; quantum dot devices.

\section{INTRODUCTION}

High power pulsed laser diode operation is desirable for multiple biomedical applications including photo-depilation [1, 2], laser liposuction [3], generation of ceramides and heat-shock proteins [4] and direct material processing [5]. In many applications, quantum dot semiconductor lasers (QDLs) have significant advantages as the high power pulse generator due to their small size and high gain efficiency [6]. In this work, the dynamical shaping of the high power pulsed operation in electrically pumped QD laser is analyzed. The output pulse shape is observed to be dependent on the pump current. At lower currents which correspond to the lasing at the ground state (GS), the pulse shape mimics that of the pump pulse. At higher currents the pulse shape becomes progressively unstable. The instability is best pronounced in the proximity of the secondary threshold which corresponds to the appearance of generation at the excited state (ES). After the slow rise stage, the output power sharply drops out. It is followed by a long-time power off stage and large-scale amplitude fluctuations. We explain the observations by the dynamic change of the linewidth enhancement factor in QD laser between the first (GS) and the secondary (ES) threshold and reveal the role of the slowly rising pumping processes in the pulse shaping and power dropouts at higher currents. 
Slowly varying control parameters may significantly affect the dynamics at the bifurcation points. The bifurcation is delayed compared to adiabatic conditions due to the inertia in the system's response [7], what results in multiple phenomena including jump transitions and memory effects. Importance of the slow passage in the construction of the bifurcation diagram led to the vast mathematical literature, but its direct experimental observation remains a complicated task since the effect of noise obstructs manifestation.

\section{EXPERIMENTAL SETUP AND SAMPLES}

Experimentally, the studied QDL structure was MBE-grown on a GaAs substrate. The active region included five layers of self-assembled InAs QDs separated with a GaAs spacer from a 5.3nm thick covering layer of $\operatorname{In}_{0.14} \mathrm{Ga}_{0.86} \mathrm{As}$. The structure was processed into $4 \mu \mathrm{m}$-wide mesa stripe devices. The 1.5 to $2.5 \mathrm{~mm}$-long lasers with high- and antireflection coatings on the rear and front facets lase either at GS $(\sim 1265 \mathrm{~nm})$ or simultaneously at GS and ES $(\sim 1190 \mathrm{~nm})$.

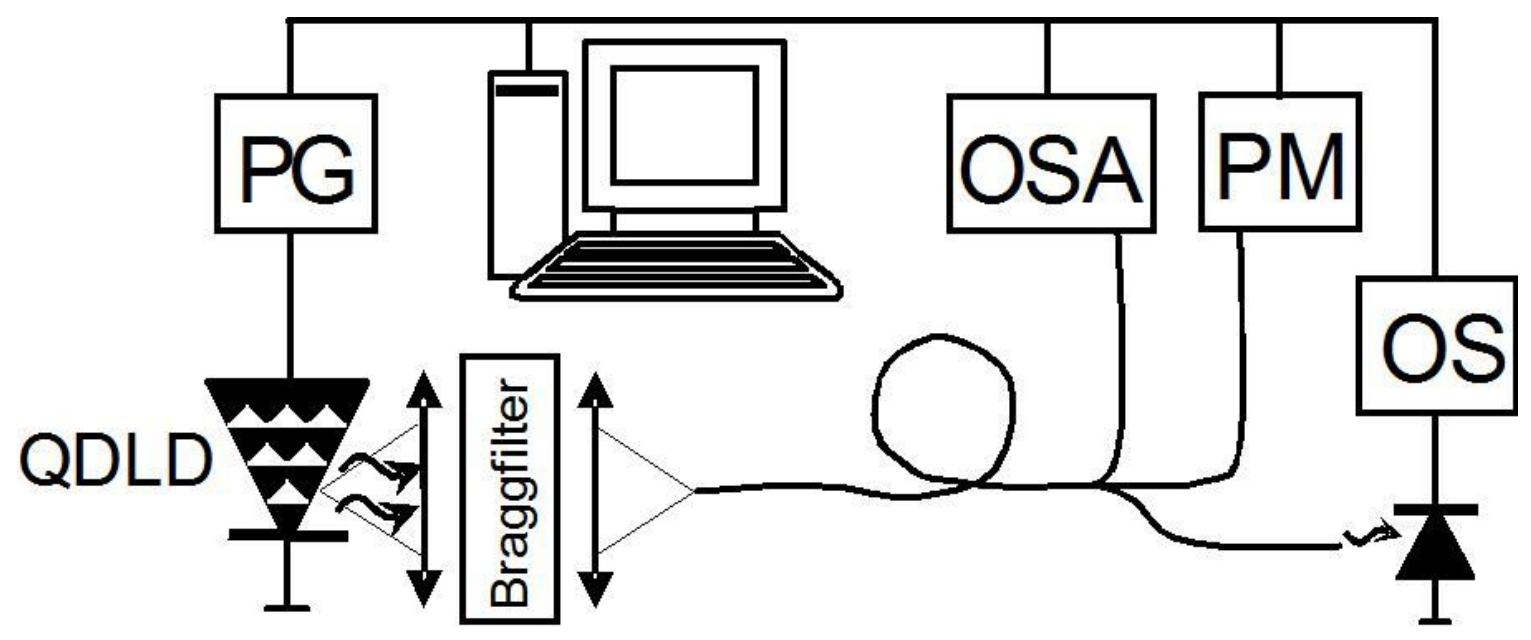

Figure 1. Schematic view of the experimental setup.

Under 30ns pulse pumping, the output pulse shape strongly depends on pumping amplitude. Such a short-pulsed electrical pumping was used to achieve high output power operation and avoid the effect of overheating on the output pulse shape. Pulses of $\sim 5 \mathrm{~ns}$ rise-time were obtained from a high power digital pulse source (up to $2 \mathrm{~A}$ current) and the laser output was detected using a high-speed pin detector with a cut-of frequency of $30 \mathrm{GHz}$ and a $50 \mathrm{GHz}$ digital oscilloscope. Schematic view of the experimental setup is shown in Fig.1 and further details of the experimental technique can be found in $[8,9]$.

\section{EXPERIMENTAL RESULTS}

The experimental output pulse shapes and corresponding optical spectra are shown in Fig. 2. We distinguish three different regimes of operation in relation to the ES threshold. In Fig. 1(a), the GS output pulse shape is similar to that of the pulsed pump up to $600 \mathrm{~mA}$ pump current. It consists of a $\sim 5 \mathrm{~ns}$ rise time, flat operation for $\sim 20 \mathrm{~ns}$, and then a $\sim 5 \mathrm{~ns}$ fall time. In Fig. 2(b), the laser operates in the proximity of the ES threshold. After the $\sim 5 \mathrm{~ns}$ rise stage, the GS output power gradually decreases. It is followed by the fast oscillations at the relaxation oscillation frequency. At higher currents (Fig. 2(c,d)), the laser output at the pulse front first corresponds to the GS. As the pump pulse reaches its top, the output power drops out. A few ns laser nearly off-stage then appears and is followed by large amplitude oscillations. The power dropout may appear at or before the rise-time of 5ns (Fig. 2(d)). To summarize the experimental results, we conclude that the pulse profile deformation and the power dropout must be related to the appearance of the lasing at ES. At lower currents, the power dropout event can either correspond to the rise-time of $5 \mathrm{~ns}$ or be slightly slower. At higher currents, the dropout is normally faster than the rise-time and indicates that the peak pump power has not yet been reached. 


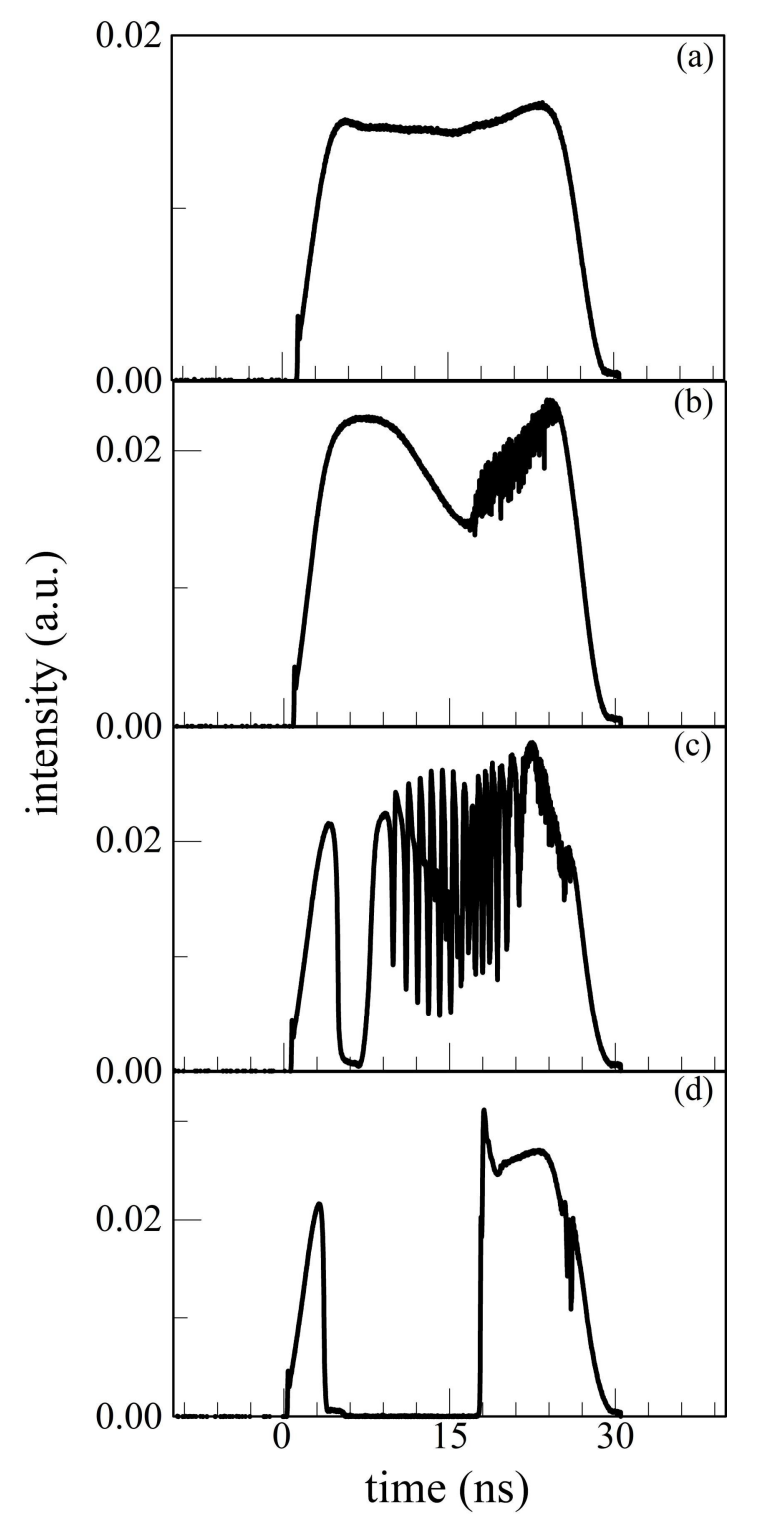

Figure 2. Experimental time traces for a range of pump currents: $800 \mathrm{~mA}$ (a), $1200 \mathrm{~mA}$ (b), $1400 \mathrm{~mA}$ (c) and $2000 \mathrm{~mA}$ (d).

The complicated patterns after dropouts are difficult to characterize and we do not discuss these dynamics focusing on the effect of the power dropout, which we address to the transformations in the phase-amplitude coupling of the field in the vicinity of the ES threshold.

\section{DISCUSSION}

In order to describe the experimental observation, we exploit the delay differential equation (DDE) model similar to [10]. The DDE approach supports multimode generation what is essential to describe the effect. Objective of the modeling is to clarify the origin of the power dropout. We, therefore, do not consider the ES in the model.

In semiconductor lasers, the phase-amplitude coupling can be conventionally characterized by the linewidth enhancement factor [11]. Its impact on semiconductor laser dynamics has been a subject of intensive research for decades. The measurements of the linewidth enhancement factor in QD laser are not so well documented. It has been, in particular, demonstrated that the various measurement techniques can lead to different values of the linewidth enhancement factor [12]. 

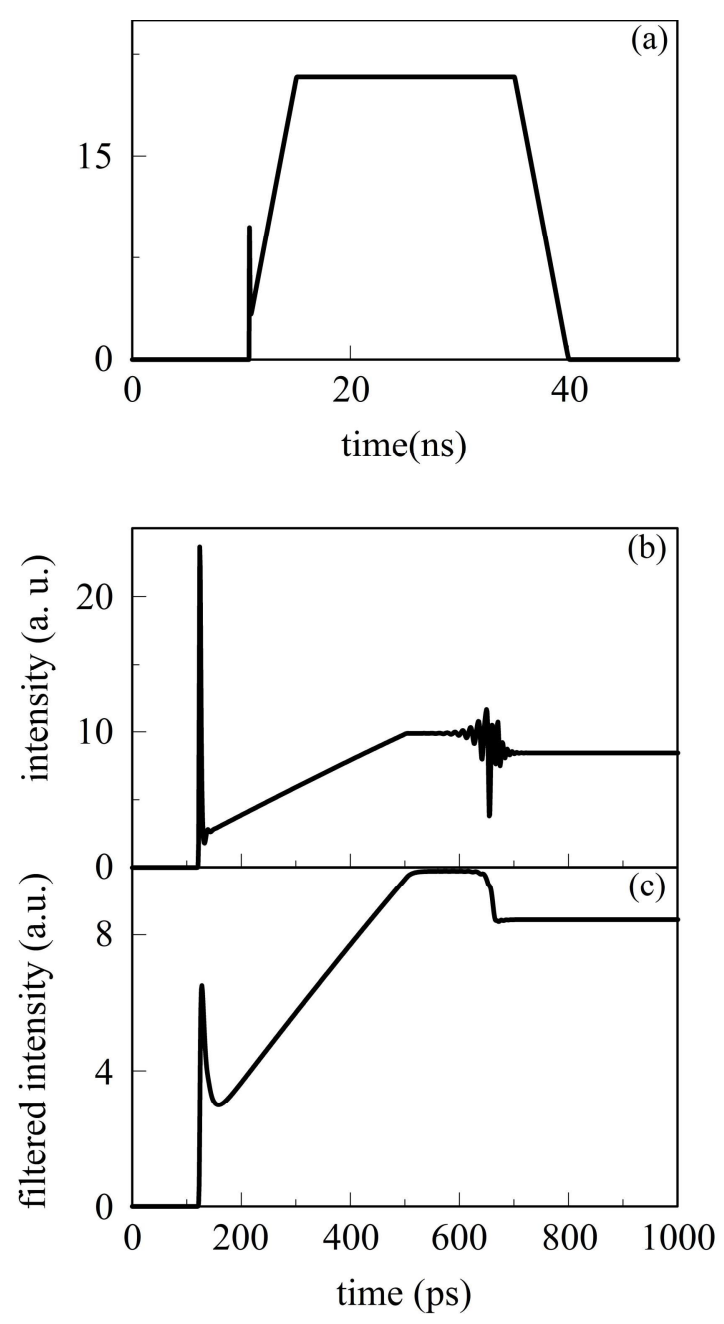

Figure 3. Numerical time traces illustrating: stable pulse profile (a); the transition from the unstable maximum gain mode to the other single mode at the increased pump current: unfiltered (c) and filtered (d).

Numerical results presented in $[13,14]$ suggest a complex dynamics of the linewidth enhancement factor in QD lasers. Experimental results for simultaneous GS/ES lasing [15], reveal that opposite to more conventional semiconductor lasers, the linewidth enhancement factor in QD lasers noticeably increases above GS threshold and a giant value 57 is measured just below the ES threshold. The increase of linewidth enhancement factor with pump current is one of the key assumptions in our modeling. The pulse pump used for our simulations accounts to $5 \mathrm{~ns}$ rise (fall) time and a plateau of $20 \mathrm{~ns}$. We consider the linewidth enhancement factor as a function of the pump current, and, therefore, it is a function of time of the pulsed pump in our case. We apply the same linear time dependence ( $5 \mathrm{~ns}$ rise time and plateau) to the linewidth enhancement factor as to the pump current.

Some examples of the filtered intensity shown in Fig. 3. In order to match the experimental results, we have filtered the time series over $30 \mathrm{GHz}$, corresponding to the bandwidth of the experimental recorder. At lower pump currents, the output pulse is similar to the pump pulse (Fig. 3a). At higher currents and, therefore, larger linewidth enhancement factors, the intensity also follows the pump temporal evolution at the front of the pulse, but either slowly decreases or sharply drops at a certain moment (Fig. 3b,c). The power dropout is followed by the fast large-amplitude oscillations. It corresponds to the experimental observations at higher currents.

Our numerical simulations reveal the following scenario for the laser output dynamics and the dropout. After the laser turns on, what can be featured by the damped fast oscillations, the laser output follows the lasing at the maximum gain 


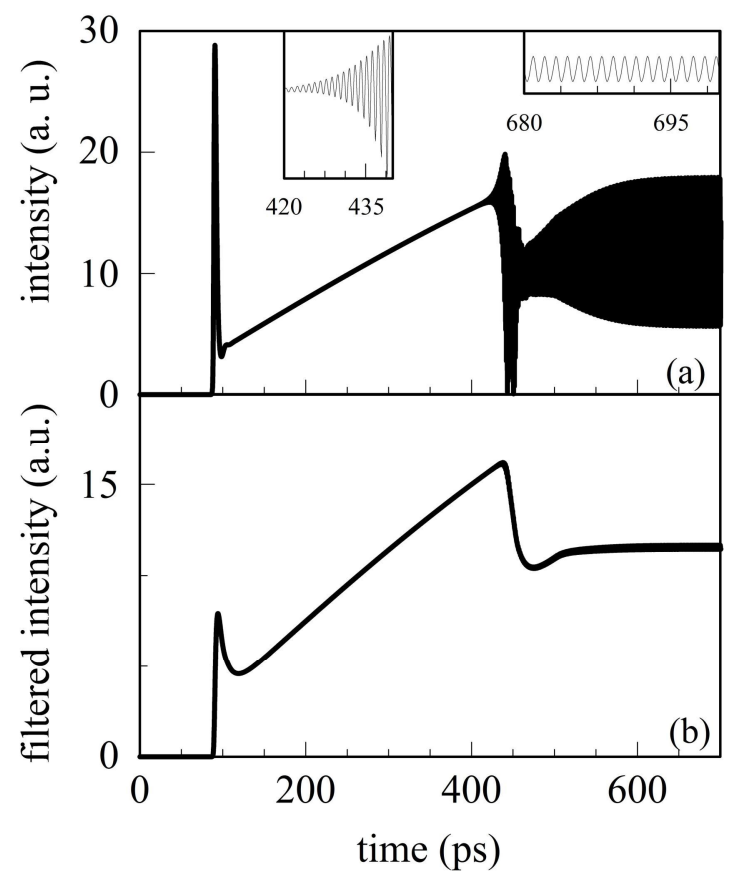

Figure 4. Numerical time traces illustrating the transition from the unstable maximum gain mode to the two mode beating: unfiltered (a) and filtered (b).

mode. At a certain value of the pump current, this mode becomes unstable, but the transition to the other mode or a complicated multimode regime is happening with a certain delay (Fig. 4a,b illustrates "single mode - two modes" transition). A slow variation of the control parameters is found to have significant effect on multiscale dynamical phenomena. In particular, the slow passage through a Hopf bifurcation is featured by a significant delay when the system changes from slowly varying steady state to slowly varying oscillation as lasing modes do not start generation simultaneously, but appear sequentially with the increase of pump. In turn, the change of the linewidth enhancement factor leads to significant shifts in the position of the lasing modes. Therefore the power dropout is explained as a sharp transition from the maximum gain mode operation to an operation with a number of modes. A chaotic itineracy of different modes with a drift is known as an effect of low frequency fluctuations in a semiconductor laser subject to optical feedback and has been a subject of intensive studies. The effect of power dropout in our work has some similar features.

\section{SUMMARY}

We demonstrate that slow rise time of the pulsed pump may affect high intensity output pulse in quantum dot laser, and lead to sharp power dropouts and deformation of the pulse profile. We explain the observation that the phase-amplitude coupling dynamicaly changes in the proximity of the ES threshold. The DDE modeling supports the experimental observations.

\section{REFERENCES}

[1] I. Greppi, "Diode laser hair removal of the black patient," Lasers in Surgery and Medicine, 28(2), 150-155 (2001).

[2] P.Chen, "Laser Liposuction Using 980-nm Diode Laser vs 1064-nm Nd:YAG for Laser Lipolysis and LipoAspiration for Male Breast Reduction (A Pioneering Largest Personal Series in Australia 2005-2009) - An Effective, Safe, Minimal Invasive Method for Treating Gynecomastia," 13th Annual Meeting of the American-Society-ofBreast-Surgeons, Phoenix, 2-6 May 2012. 
[3] H.Y.Zhang, L.Zhang, P.Tidemand-Lichtenberg, P.Buchhave, X.B.Xu, Y.X.Li, "Effect of Laser and LED on Enzymatic Production of Ceramide," Photochemistry and Photobiology, 87(1), 131-136 (2011).

[4] G.S.Sokolovskii, S.B.Onikienko, A.V.Zemlyanoi, N.A.Pikhtin, I.S.Tarasov, B.A.Margulis, I.V.Guzova, "HSP70 and Ceramide release by diode laser-treated mouse skin cells in vivo," Int. Conf. Laser Optics, St. Petersburg, Russia, 28 June - 2 July 2010.

[5] W.Qin, Y.Liu, Y.Cao, J.Gao, F.Pan, Z.Wang, "2000W high beam quality diode laser for direct materials processing," Proc. SPIE, 8197, 81971J (2011).

[6] E.U.Rafailov, M.A.Cataluna, W.Sibbett, "Mode-locked quantum-dot lasers," Nature Photonics, 1, 395-401 (2007).

[7] S. M. Baer, T. Erneux, and J Rinzel, "The Slow Passage Through a Hopf Bifurcation: Delay, Memory Effects, and Resonance,” SIAM J. Appl. Math., 49, 55 (1989).

[8] G.S.Sokolovskii, A.G.Deryagin, V.I.Kuchinskii, "Relaxation oscillations in InGaAsP/InP $(\lambda=1.55 \mu \mathrm{m})$ heterolasers with a saturable absorber", Tech.Phys.Lett., 22(4), 286 (1996).

[9] G.S.Sokolovskii, V.V.Dudelev, E.D.Kolykhalova, A.G.Deryagin, M.V.Maximov, A.M.Nadtochiy, V.I.Kuchinskii, S.S.Mikhrin, D.A.Livshits, E.A.Viktorov, T.Erneux, "Nonvanishing turn-on delay in quantum dot lasers", Appl. Phys. Lett., 100, 081109 (2012).

[10]E. A. Viktorov, P. Mandel, G. Huyet, "Long-cavity quantum dot laser,” Opt. Lett., 32, 1268 (2007).

[11] C. H. Henry, "Theory of the linewidth of semiconductor lasers," IEEE J. Quantum Electron. 18(2), 259 (1982).

[12] S. Melnik, G. Huyet, A. Uskov, "The linewidth enhancement factor $\alpha$ of quantum dot semiconductor lasers," Opt. Expr., 14, 2950 (2006).

[13]E. Gehrig and O. Hess, "Dynamic amplitude-phase coupling in quantum-dot lasers," Appl. Phys. Lett. 86, 203116 (2005).

[14]B. Lingnau, K. Lüdge, W.W. Chow and E. Schöll, "Failure of the $\alpha$ factor in describing dynamical instabilities and chaos in quantum-dot lasers," Phys.Rev. E 86, 065201 (2012).

[15]B. Dagens, A. Markus, J.X. Chen, J.-G. Provost, D. Make, O. Le Gouezigou, J. Landreau, A. Fiore, B. Thedrez, "Giant linewidth enhancement factor and purely frequency modulated emission from quantum dot laser," Electron. Lett., 41, 323 (2005). 